\title{
Degeneracy and long-range correlation: A simulation study
}

\author{
Didier Delignières $^{*}$ Vivien Marmelat ${ }^{* \dagger}$ Kjerstin Torre* \\ (*) EA 2991 Movement to Health, EuroMov, University Montpellierl, France \\ (†)Research Institute MOVE, Free University Amsterdam, The Netherlands \\ E-mail:didier.delignieres@univ-montp1.fr
}

\begin{abstract}
We present in this paper a simulation study that aimed at evidencing a causal relationship between degeneracy and long-range correlations. Long-range correlations represent a very specific form of fluctuations that have been evidenced in the outcomes time series produced by a number of natural systems. Long-range correlations are supposed to sign the complexity, adaptability and flexibility of the system. Degeneracy is defined as the ability of elements that are structurally different to perform the same function, and is presented as a key feature for explaining the robustness of complex systems. We propose a model able to generate long-range correlated series, and including a parameter that account for degeneracy. Results show that a decrease in degeneracy tends to reduce the strength of long-range correlation in the series produced by the model.
\end{abstract}

\section{Introduction}

Long-range correlations have been evidenced in a number of situations. In the domain of movement sciences, this kind of fluctuation has been evidenced in stride interval series during walking and running [1, 2], in the time interval series produced in finger tapping or in forearm oscillations [3], in the series of asynchronies produced in synchronized tapping [4], in the series of relative phases in bimanual coordination tasks [5].

There is now a general agreement for considering long-range correlations as reflecting the complexity of the system, defined as the flexible and adaptable coordination between its multiple components and subsystems [6]. Long-range correlations are supposed to sign an optimal compromise, between order and disorder, order reflecting a too strict and rigid coordination, and disorder the absence of coordination [7]. Long-range correlations are considered the signature of health and adaptability, and deviations towards order and disorder have been described in elderly or pathological populations [8].
Another conceptual framework has tried to draw a link between complexity, adaptability, and health, especially through the concepts of redundancy and degeneracy [9]. Redundancy is an important property of complex systems. In this most basic meaning, redundancy signifies that similar components within the system have similar functionality. As such, redundant components can be used to replace components that fail, or can be alternatively used for achieving a given function. One can easily conceive that redundancy provides the system with stability and robustness facing external perturbations.

The concept of degeneracy is often preferred to the classical concept of redundancy for analyzing the design principles of biological systems [e.g., 9; 10]. Degeneracy refers to a partial overlap in the functions of the multiple components within the system. In degenerate systems, distinct components can perform similar functions under certain conditions, but can also assume distinct roles in others conditions. This concept of degeneracy is more adapted to the most recent definitions of complexity, suggesting that complex systems are characterized, beyond their multiple components, by a multiscaled and hierarchical structure [11].

The behavior of a complex, degenerate and multiscaled system, in a given situation, cannot be considered as resulting from the performance of a fixed and immutable network. Throughout repetitions, diverse sets of components can be recruited for achieving a given function [12]. From this point of view, variability does not result from perturbations within the system, but expresses its inherent complexity.

These two lines of reasoning suggest a possible link between long-range correlations and degeneracy. One can suppose that complexity generates typical patterns of serial dependence in the outcomes of the systems, and especially long-range correlations [7, 13, 14]. More precisely, the strength of long-range correlations in a series of outcomes could be related to the level of degeneracy that characterizes the system.

This is an Open Access article distributed under the terms of the Creative Commons Attribution-Noncommercial License 3.0, which permits unrestricted use, distribution, and reproduction in any noncommercial medium, provided the original work is properly cited. 


\section{A model of degenerative system}

We recently analyzed the fractal properties of a quite simple model, which seemed able to produced long-range correlated series [15]. This model was initially developed by West and Scafeta [16], in the domain of locomotion. Consider the following secondorder differential equation:

$$
\ddot{x}=\alpha \dot{x}-\beta \dot{x} x^{2}-\gamma \dot{x}^{3}-\omega_{i}^{2} x+Q \sqrt{\xi_{t}}
$$

In this equation the stiffness parameter $\omega_{i}^{2}$ is cycle dependent. The dynamics of this parameter is governed by the so-called "hopping model": The key element of this model is a linear Markov process $\delta_{j}$, generated by a first-order auto-regressive equation:

$$
\delta_{j}=\phi \delta_{j-1}+\eta \varepsilon_{j}
$$

where $0<\phi<1$ is a constant, and $\varepsilon_{j}$ a white noise process with zero mean and unit variance. This process could be conceived as a chain of alternative networks in the system, neighboring networks sharing common components and being then mutually correlated. This chain then contains "correlated zones" of typical size $r$ :

$$
r=-1 / \log \phi
$$

The alternative networks in the system are supposed to be successively activated by a random walk along the chain, whose jump sizes follow a Gaussian distribution of width $\rho$ (Figure 1). This random walk generates a series $\delta_{i}$, representing the networks activated at each successive iteration. In this process, correlations within the $\delta_{i}$ series increase as the size of correlation within the chain $(r)$ increases, and decrease as the width $\rho$ of the distribution of jumps increases.

The frequency of the limit cycle (Eq. 1) is determined, for each successive cycle $i$, by

$$
\omega_{i}=\omega_{0}+\mu \delta_{i}
$$

where $\omega_{0}$ represents the baseline frequency.

In the initial formulation of the model, the Markov chain was conceived as possessing an infinite length. One can considered that the length of this chain represents the number of alternative networks susceptible to satisfy the task at hand, and then provides an index of the degeneracy of the system. So we introduced a new variable in the model, $p$, corresponding to the length of the Markov chain over which the jumps are performed. The chain is then bounded by two limits, $\delta_{1}$ and $\delta_{p}$. In order to allow the model to work despite this limited range, we just reversed the direction of the jump when one or the other limit of the chain was reached.

\section{Method}

We simulated a set of time series with this model, setting the parameters to the following values: $\alpha=0.5$, $\beta=1.0, \gamma=0.02, \omega_{0}=4 \pi, Q=0.1, r=40, \eta=0.1, \rho=$ 20 , and $\mu=1.0$. These values were previously shown to produce long-range correlated series with the initial model (with a chain of infinite length). The range $p$ was systematically varied from 10 to 200 , by steps of 10. 100 series of 1024 data points were generated for each range value.

We applied the ARMA/ARFIMA modelling procedure proposed by Torre et al. [17], in order to test for the effective presence of long-range correlations in the series. The method consists in fitting 18 models to the studied series. Nine of these models are ARMA $(p, q)$ models, $p$ and $q$ varying systematically from 0 to 2 . These ARMA models do not contain any long-range serial correlation. The other nine models are the corresponding ARFIMA $(p, d, q)$ models, differing from the previous ARMA models by the inclusion of the fractional integration parameter $d$ representing

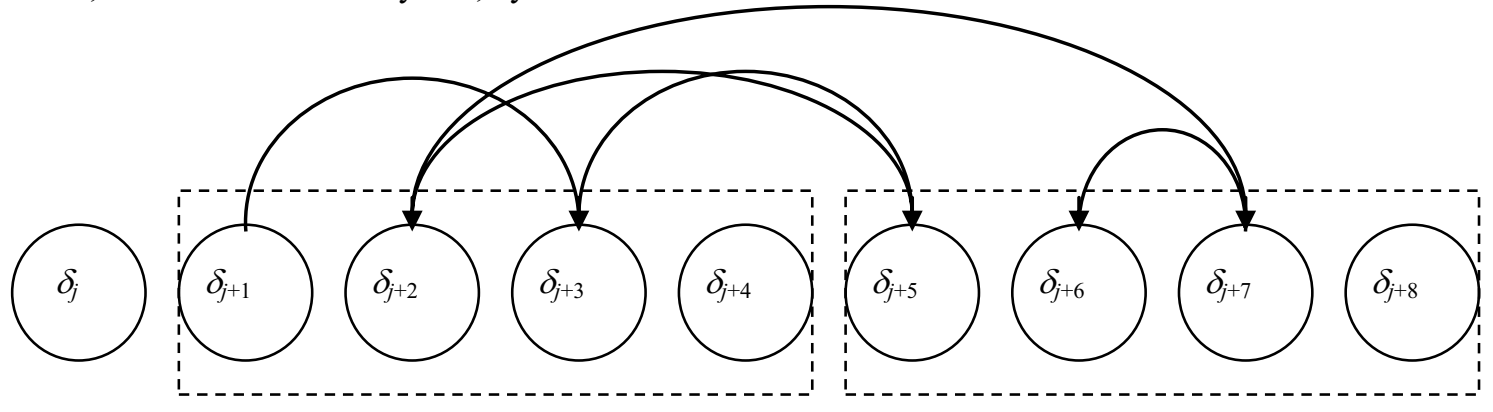

Figure 1: Illustration of the hopping model. The random walk selects successively the variables $\delta_{j+1}$, $\delta_{j+3}, \delta_{j+5}, \delta_{j+2}, \delta_{j+7}$, and $\delta_{j+6}$. The dashed boxes indicated the size (here $r=4$ ) of the correlated zones. 
persistent serial correlations. One supposes that if the series contains long-range dependence, ARFIMA models should present a better fit than the transient ARMA models.

In a second step we measured correlations in the series with the Detrended Fluctuation Analysis [18]. This method provides an exponent $\alpha$ which represents a measure of the strength of serial correlations in the series. $1 / f$ fluctuations are characterized by $\alpha$ exponents close to 1 , and uncorrelated series by exponents close to 0.5 .

\section{Results}

We present in Figure 2 some example series produced by the model, for various values of $p$. As can be seen, the series produced with high $p$ values tend to present multiple interpenetrated trends, while series produced with low $p$ values appear more stationary.
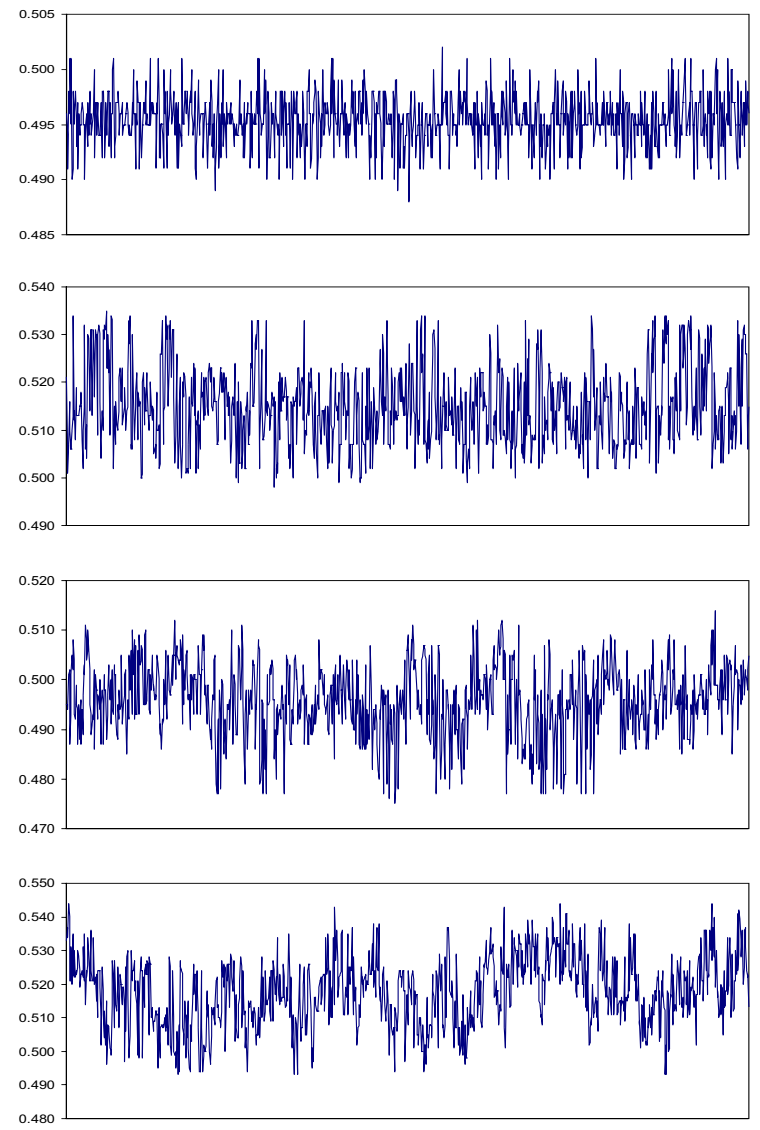

Figure 2: Example series produced by the model. From top to bottom, $p=200, p=100, p=50$, and $p=10$.
We present in Figure 3 the results of ARFIMA modeling. For range value above 60 , the percentage of series recognized as long range correlated fluctuates between 80 and $90 \%$. For lower range values, the percentage of long-range correlated series dramatically decreases.

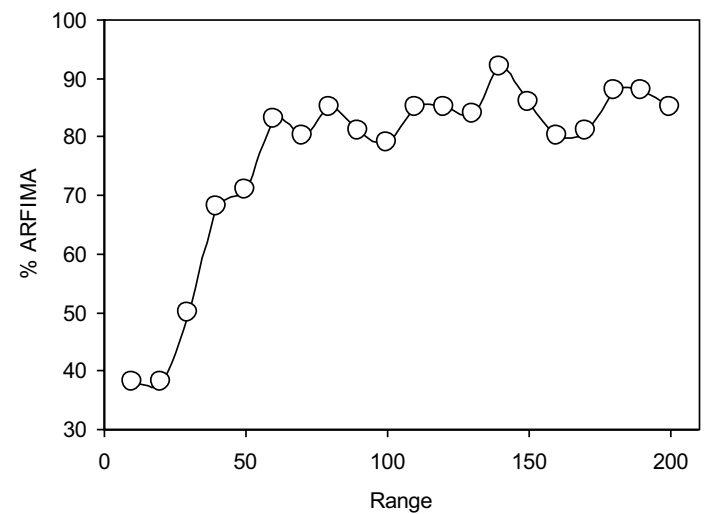

Figure 3: Percentage of series best modeled by ARMIFA models.

The results of DFA are illustrated in Figure 4. As can be seen, the model produced long-range correlated series, with $\alpha$ exponents close to 0.9 , for high $p$ values (100 and 200). Then $\alpha$ tends to decrease as $p$ decreases, and reaches values close to 0.5 for $p=20$ and $p=10$.

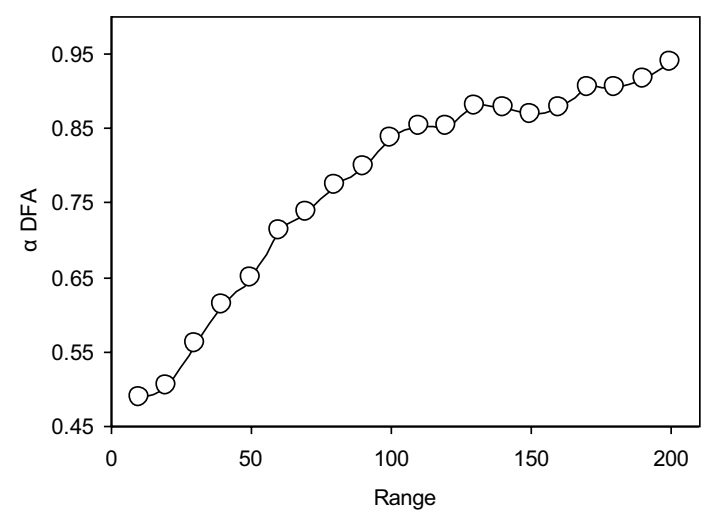

Figure 4: Evolution of the mean $\alpha$ exponent with the length of the Markov chain.

\section{Discussion}

This study suggests a direct, causal link between degeneracy and long-range correlations. Increasing degeneracy in the model induces an increase in the serial correlations in the produced series. Note, 
however, that the results obtained for the highest $p$ values in the present study are equivalent to those obtained with a chain of infinite length.

Long-range correlations tend to disappear when the length of the chain equals the theoretical length of correlated zones within the chain. In that case, one could suppose that all networks share important common components, and variability in the resulting series mainly arises from the stochastic terms in the model. Clearly, long-range correlations appear when sufficiently distinct networks are involved in the production of performance over time.

Long-range correlations and degeneracy have never been simultaneously considered. Long-range correlations characterize the series of outcomes produced by the system, but are likely to be linked to its structural and functional complexity [7,13]. Longrange correlations are supposed to characterize the outcomes of healthy and adaptive systems [8]. On the other hand, degeneracy has been evoked as a key feature for explaining the functional architecture of complex systems, and especially their properties of adaptability and robustness [9]. Considering these theoretical foundations, the present results are not surprising.

Further research is needed for investigating, in empirical studies, the implications of the present results. In particular, it seems necessary to question the nature of degeneracy, as a permanent characteristic of systems, or as a transient property, defined by the constraints of the current situation.

\section{References}

[1] J.M. Hausdorff, C.K. Peng, Z. Ladin, J.Y. Wei, and A.R. Goldberger. Is walking a random walk? Evidence for long-range correlations in stride interval of human gait. Journal of Applied Physiology, 78, 349-358, 1995.

[2] K. Jordan, J.H. Challis, J.H., and K.M. Newell. Long range correlations in the stride interval of running. Gait \& Posture, 24, 120-125, 2006.

[3] D. Delignières, L. Lemoine, and K. Torre. Time intervals production in tapping and oscillatory motion. Human Movement Science, 23, 87-103, 2004.

[4] K. Torre, and D. Delignières. Unraveling the finding of $1 / \mathrm{f}^{\beta}$ noise in self-paced and synchronized tapping: A unifying mechanistic model. Biological Cybernetics, 99 , 159-170, 2008.

[5] K. Torre, D. Delignières, and L. Lemoine. $1 / f^{\beta}$ fluctuations in bimanual coordination: An additional challenge for modeling. Experimental Brain Research, 183, 225-234, 2007.

[6] A. Diniz, M.L. Wijnants, K. Torre, J. Barreiros, N. Crato, A.M.T. Bosman, F. Hasselman, R.F.A. Cox, G.C. Van Orden, and D. Delignières. Contemporary theories of $1 / f$ noise in motor control. Human Movement Science, in press.

[7] C.T. Kello, B.C. Beltz, J.G. Holden, and G.C. Van Orden. The emergent coordination of cognitive function. Journal of Experimental Psychology: General, 136, 551-568, 2007.

[8] A.L. Goldberger, L.A.N. Amaral, J.M. Hausdorff, P.Ch. Ivanov, C.-K. Peng, and H.E. Stanley. Fractal dynamics in physiology: Alterations with disease and aging. PNAS, 99, 2466-2472., 2002.

[9] G.M. Edelman, and J.A. Gally (2001). Degeneracy and complexity in biological systems. PNAS, 98, 1376313768, 2001.

[10] G. Tononi, O. Sporns, and G.M. Edelman. Measures of degeneracy and redundancy in biological networks. PNAS, 96, 3257-3262, 1999.

[11] J. M. Carlson and J. Doyle. Complexity and robustness. PNAS, 99, 2538-2545, 2002.

[12] J.M. Whitacre, and A. Bender. Degeneracy: a design principle for robustness and evolvability Journal of Theoretical Biology, 263, 143-153, 2010.

[13] E.A.F. Ihlen, and B. Vereijken. Interaction-dominant dynamics in human cognition: Beyond $1 / f^{\alpha}$ fluctuation. Journal of Experimental Psychology: General, 139, 436-463, 2010.

[14] A.B. Slifkin, and Newell. Is variability in human performance a reflection of system noise? Current Directions in Psychological Science, 7, 170-177, 1998.

[15] D. Delignières, K. Torre, and L. Lemoine. Fractal models for event-based and dynamical timers. Acta Psychologica, 127, 382-397, 2008.

[16] B.J. West, and N. Scafetta. Nonlinear dynamical model of human gait. Physical Review E, 67, 051917, 2003.

[17] K. Torre, D. Delignières, and L. Lemoine. Detection of long-range dependence and estimation of fractal exponents through ARFIMA modeling. British Journal of Mathematical and Statistical Psychology, 60, 85-106, 2007.

[18] C.-K.Peng, S. Havlin, H.E. Stanley, and A.L. Goldberger. Quantification of scaling exponents and crossover phenomena in non stationary heartbeat time series. Chaos, 5, 82-87, 1995. 